International Mathematical Forum, 1, 2006, no. 37, 1805 - 1818

\title{
Limit cycle analysis on a cubic Hamiltonian system with quintic perturbed terms
}

\author{
Xiao-Chun Hong \\ Qujing Normal University, Qujing, Yunnan 655000 \\ People's Republic of China \\ Qing-Hua Qin ${ }^{1}$ \\ Department of Engineering, Australian National University \\ Canberra, ACT 0200, Australia
}

\begin{abstract}
This paper intends to explore bifurcation behavior of limit cycles for a cubic Hamiltonian system with quintic perturbed terms using both qualitative analysis and numerical exploration. To obtain the maximum number of limit cycles, a quintic perturbed function with the form of $R(x, y, \lambda)=S(x, y, \lambda)=m x^{2}+n y^{2}+k y^{4}-\lambda$ is added to a cubic Hamiltonian system, where $m, n, k$ and $\lambda$ are all variable. The investigation is based on detection functions which are particularly effective for the perturbed cubic Hamiltonian system. The study reveals that, for the Hamiltonian system [equation (1.5) in the introduction] with the perturbed terms mentioned above, there are 15 limit cycles if $15.1149<\lambda<15.1249$; and 11 limit cycles if $15.1102<\lambda<15.1149$. As numerical illustration, we numerically predict the detection curves and display graphically the distribution of limit cycles for the proposed perturbed Hamiltonian system.
\end{abstract}

Keywords: limit cycle, Hamiltonian system, perturbed term Mathematics Subject Classification: 34C07, 37G15, 37J40

\footnotetext{
${ }^{1}$ Corresponding author
} 


\section{Introduction}

The bifurcation of limit cycles in Hamiltonian system has long been of research interests in the circle of applied mathematics[1-6]. In particular, the bifurcation of limit cycles of the following planar polynomial system

$$
\frac{d x}{d t}=P_{n}(x, y), \quad \frac{d y}{d t}=Q_{n}(x, y) .
$$

has been considerably investigated during the past years and has now become a very popular topic in the area of applied mathematics, where $P_{n}$ and $Q_{n}$ are two polynomials of degree $\mathrm{n}$. It should be mentioned that the system (1.1) is related to the celebrated Hibert's 16th problem. For perturbed cubic Hamiltonian systems, which is extensively studied in the applied mathematical circle in comparison with other degrees of $\mathrm{n}$ of the system (1.1). For example, Liu and Li [7] considered a class of cubic Hamiltonian system perturbed with four centers and presented explicit formulas for the global and local bifurcations of the flow. Zang et al [8] studied the number of limit cycles for a cubic Hamiltonian system under quartic perturbation. Zhang et al [4] analyzed the number of limit cycles for a different cubic Hamiltonian system with cubic perturbation.

For perturbed cubic Hamiltonian system

$$
\frac{d x}{d t}=y\left(1-c y^{2}\right)-\mu x R(x, y, \lambda), \quad \frac{d y}{d t}=-x\left(1-a x^{2}\right)-\mu y S(x, y, \lambda) .
$$

where $a, c, \mu$ and $\lambda$ are real parameters, $a>0, c>0,0<\mu \ll 1, R(x, y, \lambda)$ and $S(x, y, \lambda)$ are two polynomials. Among these studies, Li and Huang [9] showed that the system (1.2) has 11 limit cycles when $R(x, y, \lambda)=S(x, y, \lambda)=$ $m x^{2}+n y^{2}-\lambda$, and obtained the Hilbert number $H(3) \geq 11$. Further, Hong and Liu [10] showed that the system (1.2) has 14 limit cycles when $R(x, y, \lambda)=$ $S(x, y, \lambda)=m x^{k}+n y^{k}-\lambda, k=10,12,14$.

For the perturbed Hamiltonian system which is slightly different from the system (1.2)

$$
\left\{\begin{array}{l}
\frac{d x}{d t}=y\left(1+x^{2}-a y^{2}\right)-\mu x R(x, y, \lambda), \\
\frac{d y}{d t}=-x\left(1-c x^{2}+y^{2}\right)-\mu y S(x, y, \lambda) .
\end{array}\right.
$$

where $a, c, \mu(0<\mu \ll 1)$ and $\lambda$ are real parameters, $R(x, y, \lambda)$ and $S(x, y, \lambda)$ are polynomials, the work of $\mathrm{Li}$ and $\mathrm{Liu}[11]$ showed that the system above has 11 limit cycles when $R(x, y, \lambda)=S(x, y, \lambda)=m x^{2}+n y^{2}-\lambda$ and obtained the 
Hilbert number $H(3) \geq 11$. Cao et als study [3] indicated that this system has 13 limit cycles when $R(x, y, \lambda)=S(x, y, \lambda)=m x^{6}+n y^{6}-\lambda$. Further, Tang and Hong [12] found that the system (1.3) has 14 limit cycles when $R(x, y, \lambda)=$ $S(x, y, \lambda)=m x^{8}+n y^{8}-\lambda$. Zhang et al [13] also explored the number of limit cycles for the Hamiltonian system of (1.3) under quartic perturbations. Wu et al [6] also explored the number of limit cycles for the Hamiltonian system of (1.3) under quintic perturbations.

For the Hamiltonian system

$$
\frac{d x}{d t}=y\left(1+x^{2}+a y^{2}\right), \quad \frac{d y}{d t}=x\left(1-c x^{2}-y^{2}\right) .
$$

Zhang et al [14] showed that the system (1.4) has 11 limit cycles under cubic perturbations. Zhang et al [8] showed that the system (1.4) has 13 limit cycles under quartic perturbations.

It is worthy noting that, based on the cubic Hamiltonian system

$$
\frac{d x}{d t}=y\left(1-x^{2}+a y^{2}\right), \quad \frac{d y}{d t}=x\left(1-c x^{2}+y^{2}\right) .
$$

where $a$ and $c$ are two real parameters and $a c>1,0<c<1, a>1$, a series of investigations on bifurcation behavior and number of limit cycles has been conduced in recent years. The work of Li and Lin [15] showed that there are 11 limit cycles for the system (1.5) under cubic perturbed terms and obtained the Hilbert number $H(3) \geq 11$. For the perturbed Hamiltonian system

$$
\frac{d x}{d t}=y\left(1-x^{2}+a y^{2}\right)-\mu x R(x, y, \lambda), \frac{d y}{d t}=x\left(1-c x^{2}+y^{2}\right)-\mu y S(x, y, \lambda) .
$$

where $a, c, \mu(0<\mu \ll 1)$ and $\lambda$ are real parameters, $R(x, y, \lambda)$ and $S(x, y, \lambda)$ are polynomials. More recently, Huang and Liu [16] constructed a quintic polynomial system with a small parameter and eight normal parameters. Zang et al[17] presented configurations of limit cycles bifurcated from a homoclinic loop for quintic systems with quintic perturbations. Chen et al [18] explored the center conditions for degenerate singular points of quintic polynomial vector field with a small parameter and eight normal parameters.

From the above review, we found that most of previous studies is focused on the $R(x, y, \lambda)=S(x, y, \lambda)=m x^{k}+n y^{k}-\lambda$. In this paper, a different perturbed function $R(x, y, \lambda)=S(x, y, \lambda)=m x^{2}+n y^{2}+k y^{4}-\lambda$, is employed to explore the bifurcation behavior and the distribution of limit cycles of the system (1.6). $m, n, k$ and $\lambda$ here, rather than $m, n$ and $\lambda$ only in the existing work, are all variable. Using the same method of detection function and method of 
numerical exploration as in $[10,12]$, the study firstly shows that the system

$$
\left\{\begin{array}{l}
\frac{d x}{d t}=y\left(1-x^{2}+a y^{2}\right)-\mu x\left(m x^{2}+n y^{2}+k y^{4}-\lambda\right), \\
\frac{d y}{d t}=x\left(1-c x^{2}+y^{2}\right)-\mu y\left(m x^{2}+n y^{2}+k y^{4}-\lambda\right) .
\end{array}\right.
$$

has 15 limit cycles when $a=6, c=0.25, m=2, n=-13.47, k=2.3,0<$ $\mu \ll 1,15.1149<\lambda<15.1249$, and 11 limit cycles if $15.1102<\lambda<15.1149$.

\section{Detection function and detection curves}

In this section some preliminary results on the detection functions for the perturbed Hamiltonian system (1.7) are briefly reviewed in order to provide a common source of formulation for the analysis in later sections. Let us begin with considering the perturbed Hamiltonian system.

Ye [2] obtained some useful results by introducing.

Lemma 1. Consider the perturbed Hamiltonian system

$$
\frac{d x}{d t}=-\frac{\partial H}{\partial y}+P(x, y, \alpha), \quad \frac{d y}{d t}=\frac{\partial H}{\partial x}+Q(x, y, \alpha) .
$$

and the corresponding unperturbed Hamiltonian system

$$
\frac{d x}{d t}=-\frac{\partial H}{\partial y}, \quad \frac{d y}{d t}=\frac{\partial H}{\partial x}
$$

Obviously system (2.1) reduces into system $(2.2)$ when $P(x, y, 0) \equiv Q(x, y, 0)$ $\equiv 0$. The curve $\Gamma^{h}$ defined by Hamiltonian $H(x, y)=h$ of system $(2.2)$ is, then, closed orbits and will extend to the outside of $\Gamma^{h}$ as $h$ increases, and $\Gamma^{h}(D)$ is the area inside $\Gamma^{h}$. If there exists $h^{*}$ such that the function

$$
A(h)=\iint_{\Gamma^{h}(D)}\left[P_{x \alpha}^{\prime \prime}(x, y, 0)+Q_{y \alpha}^{\prime \prime}(x, y, 0)\right] d x d y .
$$

satisfies $A\left(h^{*}\right)=0$ and $A^{\prime}\left(h^{*}\right) \neq 0, \alpha A^{\prime}\left(h^{*}\right)<0(>0)$, then system (2.1) has only one stable (unstable) limit cycle near to $\Gamma^{h^{*}}$ when $\alpha$ is vary small. Conversely, If $\Gamma^{h}$ is constrained inside as $h$ increases, the stable properties of the limit cycle are opposite of that described above, i.e., when $\alpha A^{\prime}\left(h^{*}\right)<0(>0)$ the limit cycle is unstable (stable). If $A(h) \neq 0$, then system $(2.1)$ has no limit cycle.

Li and Li [19] considered the following system:

$$
\frac{d x}{d t}=-\frac{\partial H}{\partial y}-\mu x[p(x, y)-\lambda], \quad \frac{d y}{d t}=\frac{\partial H}{\partial x}-\mu y[q(x, y)-\lambda] .
$$


where $p(0,0)=q(0,0)=0$. Using the results given in [2] above, it follows from $A(h)=0$ that

$$
\lambda=\lambda(h)=\frac{\iint_{\Gamma^{h}(D)} f(x, y) d x d y}{2 \iint_{\Gamma^{h}(D)} d x d y},
$$

where

$$
f(x, y)=x p_{x}^{\prime}+y q_{y}^{\prime}+p+q .
$$

The function $\lambda(h)$ is usually known as the detection function of system (2.4). Using the detection function $\lambda(h)$ and lemma 1 above, the following proposition regarding the limit cycle of system (2.4) can be obtained [20]:

Proposition 1. For any given $\lambda_{0}$ : (1) If $\left(h_{0}, \lambda\left(h_{0}\right)\right)$ is an intersecting point of the line $\lambda=\lambda_{0}$ and the detection curve $\lambda=\lambda(h)$, and $\lambda^{\prime}\left(h_{0}\right)>0(<0)$, then the system (2.4) has only one stable (unstable) limit cycle near $\Gamma^{h_{0}}$ when $\lambda=\lambda_{0} ;(2)$ If line $\lambda=\lambda_{0}$ and the detection curve $\lambda=\lambda(h)$ have no intersecting point, then system (2.4) has no limit cycle when $\lambda=\lambda_{0}$. Conversely, If $\Gamma^{h}$ is constrained inside as $h$ increases, the stability of the limit cycle is opposite to the results above.

The proof of this proposition can be found elsewhere [12]. For the sake of completeness, we briefly present the proof as below:

Proof. In Lemma 1 , let $\alpha=-\mu, P(x, y, \alpha)=\alpha x[p(x, y)-\lambda], Q(x, y, \alpha)=$ $\alpha y[q(x, y)-\lambda]$. We have $P(x, y, 0) \equiv Q(x, y, 0) \equiv 0$ and $P_{x \alpha}^{\prime \prime}+Q_{y \alpha}^{\prime \prime}=f(x, y)-$ $2 \lambda$. Thus the $A(h)$ in Lemma 1 becomes

$$
A(h)=\iint_{\Gamma^{h}(D)} f(x, y) d x d y-\lambda \iint_{\Gamma^{h}(D)} 2 d x d y .
$$

By denoting $\psi(h)=\iint_{\Gamma^{h}(D)} f(x, y) d x d y$ and $\phi(h)=\iint_{\Gamma^{h}(D)} 2 d x d y$, we have $\lambda=\frac{\psi\left(h_{0}\right)}{\phi\left(h_{0}\right)}$, from $A\left(h_{0}\right)=0$, which leads to

$$
A^{\prime}\left(h_{0}\right)=\psi^{\prime}\left(h_{0}\right)-\lambda \phi^{\prime}\left(h_{0}\right)=\frac{\psi^{\prime}\left(h_{0}\right) \phi\left(h_{0}\right)-\psi\left(h_{0}\right) \phi^{\prime}\left(h_{0}\right)}{\phi\left(h_{0}\right)} .
$$

We also have from (2.5) that

$$
\lambda^{\prime}\left(h_{0}\right)=\frac{\psi^{\prime}\left(h_{0}\right) \phi\left(h_{0}\right)-\psi\left(h_{0}\right) \phi^{\prime}\left(h_{0}\right)}{\left[\phi\left(h_{0}\right)\right]^{2}} .
$$

It is noted from (2.5) that $\phi\left(h_{0}\right)>0$. Moreover, both $A^{\prime}\left(h_{0}\right)$ and $\lambda^{\prime}\left(h_{0}\right)$ have the same sign by comparing (2.8) with (2.9). Therefore, $-\mu A^{\prime}\left(h_{0}\right)$ and $-\mu \lambda^{\prime}\left(h_{0}\right)$ also have the same sign. From Lemma 1, the proof of Proposition 1 is thus completed. 


\section{Limit cycles distribution of perturbed system}

Consider again the Hamiltonian systems (1.5). The related Hamiltonian function is assumed to be in the form

$$
H(x, y)=c x^{4}+a y^{4}-2 x^{2} y^{2}+2\left(y^{2}-x^{2}\right) .
$$

Letting $H(x, y)=h$, we have

$$
c x^{4}+a y^{4}-2 x^{2} y^{2}+2\left(y^{2}-x^{2}\right)=h .
$$

In polar coordinate system, from (1.5), we get

$$
\left\{\begin{array}{l}
\frac{d r}{d t}=\frac{1}{4} r \sin (2 \theta)\left\{4+[(a+c)-(a-c+2) \cos (2 \theta)] r^{2}\right\}, \\
\frac{d \theta}{d t}=-\frac{1}{4}\left[(a+c-2)-2(a-c) \cos (2 \theta)+(a+c+2) \cos ^{2} \theta\right] r^{2}+\cos (2 \theta) .
\end{array}\right.
$$

From (3.1) and (3.2), we get

$$
H(r, \theta)=r^{4} u(\theta)-2 r^{2} \cos (2 \theta)=h .
$$

where

$$
u(\theta)=a \sin ^{4} \theta+c \cos ^{4} \theta-2 \sin ^{2} \theta \cos ^{2} \theta .
$$

It follows from (3.4) that:

$$
R_{ \pm}=r_{ \pm}^{2}(\theta, h)=\frac{\cos (2 \theta) \pm \sqrt{v(\theta)}}{u(\theta)} .
$$

where

$$
v(\theta)=\cos ^{2}(2 \theta)+h u(\theta) .
$$

Letting $\frac{d \theta}{d t}=0$ in $(3.3)_{2}$, it follows that:

$$
\theta_{ \pm}(h)=\frac{1}{2} \arccos \left[\frac{a-c \pm \sqrt{(a-c)^{2}-(a+c-2)\left(a+c+2+4 h^{-1}\right)}}{a+c+2+4 h^{-1}}\right] .
$$

The curves defined by (3.1) or (3.3) form the following three types of closed orbits when the parameter $h$ varies from $-\frac{a+c-2}{a c-1}$ to $+\infty$.

$$
\Gamma_{1}^{h}:-\frac{a+c-2}{a c-1}<h<-\frac{1}{c}, \quad \Gamma_{2}^{h}:-\frac{1}{c}<h<0, \quad \Gamma_{3}^{h}: 0<h<+\infty .
$$

The phase portrait of (1.5) is shown in Fig. 1. It is noted that the all curves will extend outwards as $h$ increases. 


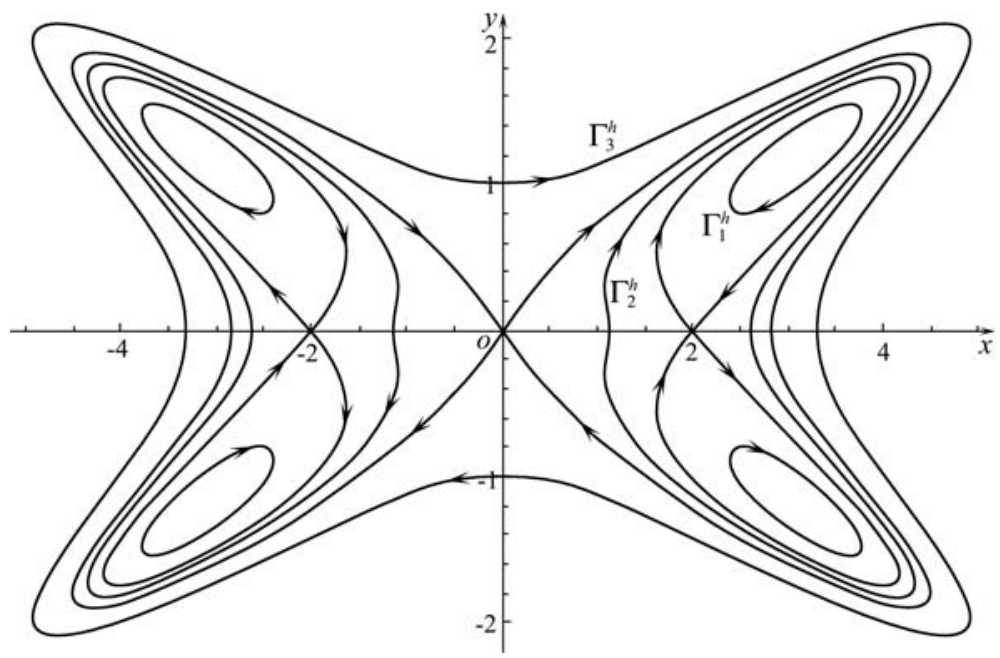

Fig. 1. Phase portrait of the unperturbed system (1.5) when $a=6$ and $c=0.25$.

Consider again the perturbed Hamiltonian system (1.7). Using (2.3), the system (1.7) has three equations

$$
\iint_{\Gamma_{j}^{h}(D)}\left(\frac{\partial^{2} P}{\partial x \partial \mu}+\frac{\partial^{2} Q}{\partial y \partial \mu}\right) d x d y=0 \quad(j=1-3) .
$$

which correspond to the three families of closed orbits $\Gamma_{j}^{h}(j=1-3)$ in Fig. 1. By comparing (1.7) with (2.1) and (2.4), we have

$$
\frac{\partial^{2} P}{\partial x \partial \mu}+\frac{\partial^{2} Q}{\partial y \partial \mu}=4 m x^{2}+4 n y^{2}+6 k y^{4}-2 \lambda .
$$

By considering (3.2)-(3.12), the detection function $\lambda_{j}(h)$ can finally be given in the form

$$
\lambda_{j}(h)=\frac{\iint_{\Gamma_{j}^{h}(D)}\left(4 m x^{2}+4 n y^{2}+6 k y^{4}\right) d x d y}{2 \iint_{\Gamma_{j}^{h}(D)} d x d y} \quad(j=1-3) .
$$

\section{Distribution of limit cycles}

For simplicity, let $a=6, c=0.25$ in (1.7). Based on (3.13)-(3.15), the three detection functions of the system (1.7) can then be expressed in the polar coordinate system as

$$
\lambda_{1}(h)=\frac{\int_{\theta_{+}}^{\theta_{-}}\left(R_{+}^{2}-R_{-}^{2}\right) \cos ^{2} \theta d \theta}{\int_{\theta_{+}}^{\theta_{-}}\left(R_{+}-R_{-}\right) d \theta} m+\frac{\int_{\theta_{+}}^{\theta_{-}}\left(R_{+}^{2}-R_{-}^{2}\right) \sin ^{2} \theta d \theta}{\int_{\theta_{+}}^{\theta_{-}}\left(R_{+}-R_{-}\right) d \theta} n
$$




$$
\begin{aligned}
+ & \frac{\int_{\theta_{+}}^{\theta_{-}}\left(R_{+}^{3}-R_{-}^{3}\right) \sin ^{4} \theta d \theta}{\int_{\theta_{+}}^{\theta_{-}}\left(R_{+}-R_{-}\right) d \theta} k, \quad\left(-\frac{a+c-2}{a c-1}<h<-\frac{1}{c}\right) . \\
\lambda_{2}(h)= & \frac{\int_{-\theta_{-}}^{\theta_{-}}\left(R_{+}^{2}-R_{-}^{2}\right) \cos ^{2} \theta d \theta}{\int_{-\theta_{-}}^{\theta_{-}}\left(R_{+}-R_{-}\right) d \theta} m+\frac{\int_{-\theta_{-}}^{\theta_{-}}\left(R_{+}^{2}-R_{-}^{2}\right) \sin ^{2} \theta d \theta}{\int_{-\theta_{-}}^{\theta_{-}}\left(R_{+}-R_{-}\right) d \theta} n \\
& +\frac{\int_{-\theta_{-}}^{\theta_{-}}\left(R_{+}^{3}-R_{-}^{3}\right) \sin ^{4} \theta d \theta}{\int_{-\theta_{-}}^{\theta_{-}}\left(R_{+}-R_{-}\right) d \theta} k, \quad\left(-\frac{1}{c}<h<0\right) . \\
\lambda_{3}(h)= & \frac{\int_{0}^{2 \pi} R_{+}^{2} \cos ^{2} \theta d \theta}{\int_{0}^{2 \pi} R_{+} d \theta} m+\frac{\int_{0}^{2 \pi} R_{+}^{2} \sin ^{2} \theta d \theta}{\int_{0}^{2 \pi} R_{+} d \theta} n+\frac{\int_{0}^{2 \pi} R_{+}^{3} \sin ^{4} \theta d \theta}{\int_{0}^{2 \pi} R_{+} d \theta} k, \\
& (0<h<+\infty) .
\end{aligned}
$$

which corresponds to the three families of closed orbits $\Gamma_{j}^{h}(j=1-3)$ in Fig. 1 , where

$$
\begin{aligned}
& R_{ \pm}=r_{ \pm}^{2}(\theta, h)=\frac{\cos (2 \theta) \pm \sqrt{v(\theta)}}{u(\theta)} . \\
& u(\theta)=a \sin ^{4} \theta+c \cos ^{4} \theta-2 \sin ^{2} \theta \cos ^{2} \theta . \\
& v(\theta)=\cos ^{2}(2 \theta)+h u(\theta) . \\
& \theta_{ \pm}(h)=\frac{1}{2} \arccos \left[\frac{a-c \pm \sqrt{(a-c)^{2}-(a+c-2)\left(a+c+2+4 h^{-1}\right)}}{a+c+2+4 h^{-1}}\right] .
\end{aligned}
$$

Table 1 lists the values of detection functions $\lambda_{j}(h)(j=1-3)$, obtained from (4.1)-(4.3), as they vary with the parameter $h$. The three detection functions of system (1.7) listed in Table 1 are also illustrated in Fig. 2.

Table 1 Values of detection functions $\lambda_{j}(h)(j=1-3), a=6, c=0.25$,

$$
m=2, n=-13.47 \text { and } k=2.3
$$




\begin{tabular}{llllll}
\hline$h$ & $\lambda_{1}(h)$ & $h$ & $\lambda_{2}(h)$ & $h$ & $\lambda_{3}(h)$ \\
\hline-8.49999 & 15.1149 & -3.99999 & 15.1252 & 0.00001 & 13.7102 \\
-8.40001 & 15.1161 & -3.92001 & 15.1320 & 0.10001 & 13.5600 \\
-8.30001 & 15.1172 & -3.90001 & 15.1326 & 0.20001 & 13.4746 \\
-7.90001 & 15.1209 & -3.89001 & 15.1326 & 0.50001 & 13.3284 \\
-7.70001 & 15.1222 & -3.88001 & 15.1327 & 1.00001 & 13.2367 \\
-7.50001 & 15.1232 & -3.87001 & 15.1328 & 1.20001 & 13.2280 \\
-7.30001 & 15.1242 & -3.80001 & 15.1319 & 1.24001 & 13.2276 \\
-7.10001 & 15.1246 & -3.70001 & 15.1278 & 1.25001 & 13.2275 \\
-6.95001 & 15.1249 & -3.50001 & 15.1134 & 1.30001 & 13.2277 \\
-6.70001 & 15.1246 & -3.30001 & 15.0926 & 1.40001 & 13.2298 \\
-6.50001 & 15.1244 & -2.90001 & 15.0359 & 1.50001 & 13.2342 \\
-6.30001 & 15.1237 & -2.70001 & 15.0008 & 1.60001 & 13.2404 \\
-5.90001 & 15.1215 & -2.50001 & 14.9616 & 2.00001 & 13.2820 \\
-5.70001 & 15.1200 & -2.30001 & 14.9176 & 2.50001 & 13.3633 \\
-5.50001 & 15.1182 & -1.90001 & 14.8151 & 3.00001 & 13.4690 \\
-5.30001 & 15.1163 & -1.70001 & 14.7557 & 3.50001 & 13.5931 \\
-4.90001 & 15.1126 & -1.50001 & 14.6899 & 4.00001 & 13.7313 \\
-4.70001 & 15.1111 & -1.30001 & 14.6170 & 4.50001 & 13.8814 \\
-4.50001 & 15.1103 & -0.90001 & 14.4442 & 5.00001 & 14.0409 \\
-4.45001 & 15.1102 & -0.70001 & 14.3395 & 5.50001 & 14.2087 \\
-4.40001 & 15.1103 & -0.50001 & 14.2178 & 6.00001 & 14.3832 \\
-4.30001 & 15.1110 & -0.30001 & 14.0701 & 7.00001 & 14.7492 \\
-4.10001 & 15.1160 & -0.10001 & 13.8728 & 8.00001 & 15.1332 \\
-4.00001 & 15.1252 & -0.00001 & 13.7103 & 9.00001 & 15.5313 \\
\hline & & & & &
\end{tabular}

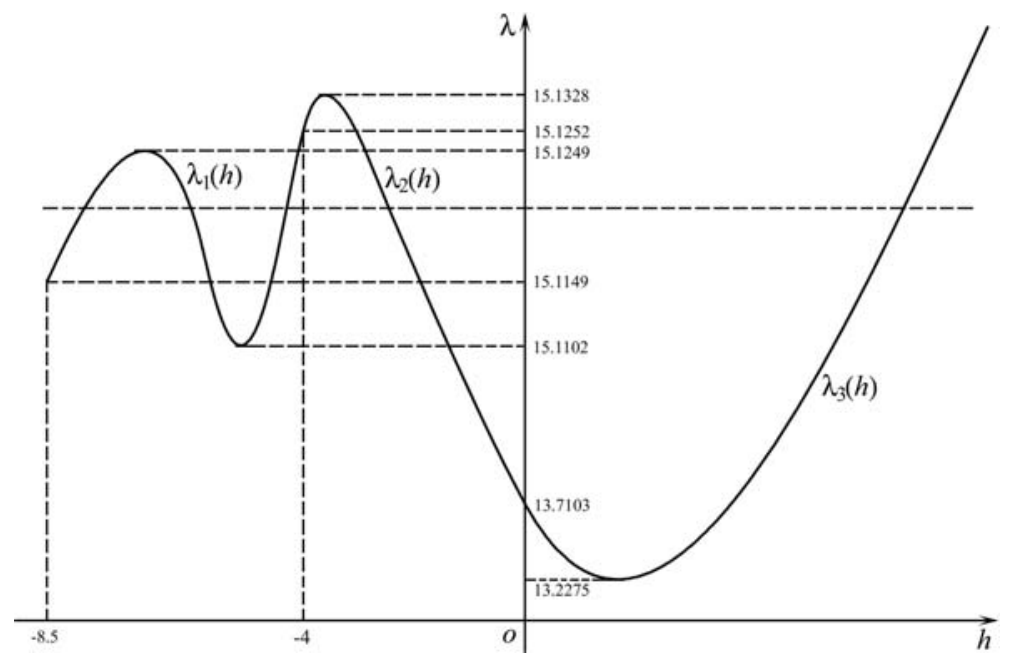

Fig. 2. Detection curves of system (1.7) when $a=6, c=0.25, m=2$, 


$$
n=-13.47 \text { and } k=2.3 \text {. }
$$

Based on the three detection curves in Fig 2 and Proposition 1 in Section 2, the following proposition can be presented:

Proposition 2 For $a=6, c=0.25, m=2, n=-13.47, k=2.3$ and $0<\mu \ll 1$, we have the following two conclusions:

(1)The system (1.7) has 15 limit cycles if $15.1149<\lambda<15.1249$ (see Fig. $3)$.

(2)The system (1.7) has 11 limit cycles if $15.1102<\lambda<15.1149$ (see Fig. 4).

It should be mentioned that other results can be similarly obtained in addition to the two cases listed above. But we omit those details for conciseness.

Figures 3 and 4 display the position of each limit cycle obtained by using the numerical exploration method [21]. In the calculation, $\lambda=15.12, \lambda=15.114$ are used.

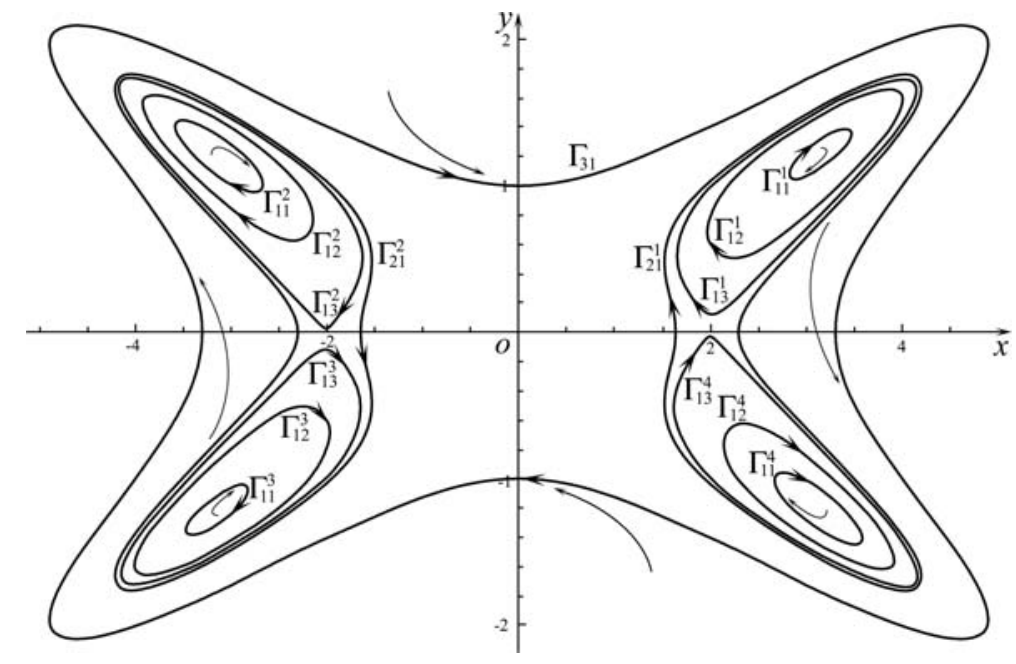

Fig. 3. The 15 limit cycles and their distribution for system (1.7) when $a=6$, $c=0.25, m=2, n=-13.47, k=2.3, \lambda=15.12$ and $\mu=0.0001$. Each limit cycle passes a particular point listed in Table 2 . 
Table 2 Points passed by limit cycles in Fig. 3.

\begin{tabular}{llll}
\hline limit cycles & passing point & limit cycles & passing point \\
\hline$\Gamma_{31}$ (stable) & $(0,0.9988)$ & $\Gamma_{12}^{1}$ (unstable) & $(3,0.7900)$ \\
$\Gamma_{21}^{1}$ (unstable) & $(1.6437,0)$ & $\Gamma_{12}^{2}$ (unstable) & $(-3,0.8397)$ \\
$\Gamma_{21}^{2}$ (unstable) & $(-1.6437,0)$ & $\Gamma_{12}^{3}$ (unstable) & $(-3,-0.7900)$ \\
$\Gamma_{11}^{1}$ (stable) & $(3,1.0608)$ & $\Gamma_{12}^{4}$ (unstable) & $(3,-0.8397)$ \\
$\Gamma_{11}^{2}$ (stable) & $(-3,1.0081)$ & $\Gamma_{13}^{1}$ (stable) & $(3,0.6955)$ \\
$\Gamma_{11}^{3}$ (stable) & $(-3,-1.0608)$ & $\Gamma_{13}^{2}$ (stable) & $(-3,0.6896)$ \\
$\Gamma_{11}^{4}$ (stable) & $(3,-1.0081)$ & $\Gamma_{13}^{3}$ (stable) & $(-3,-0.6955)$ \\
& & $\Gamma_{13}^{4}$ (stable) & $(3,-0.6896)$ \\
\hline
\end{tabular}

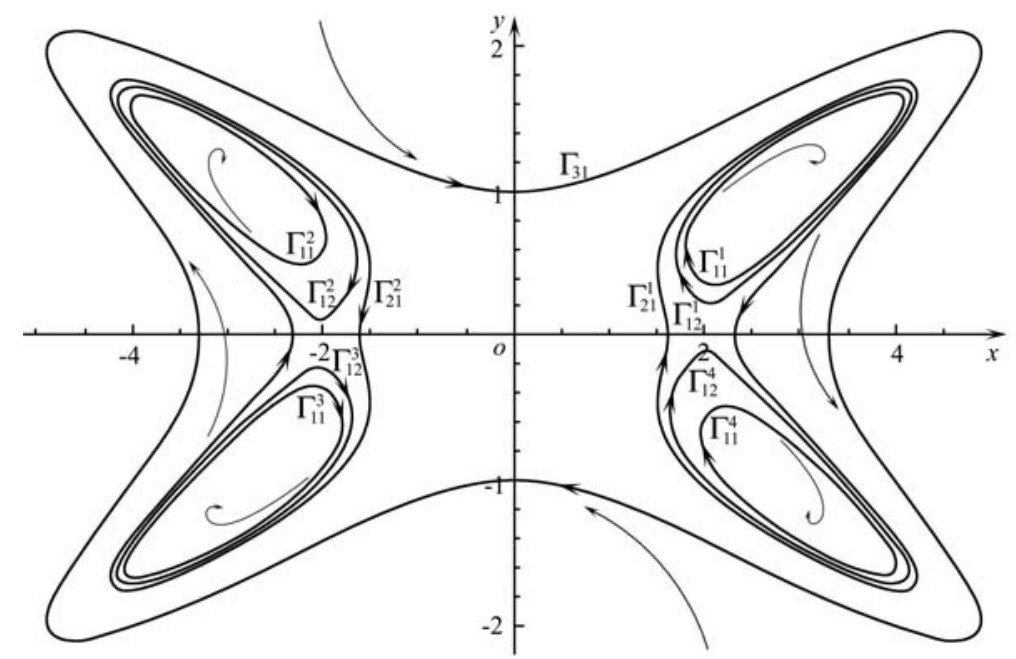

Fig. 4. The 11 limit cycles and their distribution for system (1.7) when $a=6$, $c=0.25, m=2, n=-13.47, k=2.3, \lambda=15.114$ and $\mu=0.0001$. Each limit cycle passes a particular point listed in Table 3 .

Table 3 Points passed by limit cycles in Fig. 4 .

\begin{tabular}{llll}
\hline limit cycles & passing point & limit cycles & passing point \\
\hline$\Gamma_{31}$ (stable) & $(0,0.9982)$ & $\Gamma_{11}^{4}$ (unstable) & $(3,-0.7853)$ \\
$\Gamma_{21}^{1}$ (unstable) & $(1.6126,0)$ & $\Gamma_{12}^{1}$ (stable) & $(3,0.7125)$ \\
$\Gamma_{21}^{2}$ (unstable) & $(-1.6126,0)$ & $\Gamma_{12}^{2}$ (stable) & $(-3,0.6947)$ \\
$\Gamma_{11}^{1}$ (unstable) & $(3,0.7391)$ & $\Gamma_{12}^{3}$ (stable) & $(-3,-0.7125)$ \\
$\Gamma_{11}^{2}$ (unstable) & $(-3,0.7853)$ & $\Gamma_{12}^{4}$ (stable) & $(3,-0.6947)$ \\
$\Gamma_{11}^{3}$ (unstable) & $(-3,-0.7391)$ & & \\
\hline
\end{tabular}




\section{Conclusions}

In the above discussion, we use both qualitative and numerical methods to investigate the number and distribution of limit cycles in a cubic Hamiltonian system with quintic perturbed term (1.7). The study is based on assuming that $a c>1,0<c<1, a>1,0<\mu \ll 1, m, n, k$ and $\lambda$ are all real parameters in (1.7). In particular, for $a=6, c=0.25, m=2, n=-13.47, k=2.3$ and $0<\mu \ll 1$, the study reveals that the system (1.7) has 15 limit cycles if $15.1149<\lambda<15.1249$ and has 11 limit cycles if $15.1102<\lambda<15.1149$. It is also found that each limit cycle passes a particular point and the position of these points is obtained by using the numerical exploration method [21] when $a=6, c=0.25, m=2, n=-13.47, k=2.3$ and $\mu=0.0001$ for two particular values of parameter $\lambda(15.12$ and 15.114).

\section{Acknowledgements}

This work was financially supported by the Australian Research Council (Grant No. DP0209487) and the Natural Science Foundation of China (Grant No. 10261008) and Yunnan Province (Grant No. 2005A0080M-1).

\section{References}

[1] Bautin NN. On the number of limit cycles which appear with the variation of coefficients from an equilibrium position of focus or centre type. Mat. Sb.(N.S.) 30(1952): 181-196, Amer. Math. Soc. Transl. 100 (1954).

[2] Ye YQ. The theory of limit cycles. Trans Math Monographs (Amer Math Soc) 1986: 66 .

[3] Cao HJ, Liu ZR, Jing ZJ. Bifurcation set and distribution of limit cycles for a class of cubic Hamiltonian system with higher-order perturbed terms. Chaos, Solitons and Fractals, 2000; 11: 2293-2304.

[4] Zhang TH, Han MA, Zang H. Perturbation from an asymmetric cubic Hamiltonian. J. Math. Anal. Appl., 2005; 305: 617-630.

[5] Wang S, Yu P. Bifurcation of limit cycles in a quintic Hamiltonian system under a sixth-order perturbation. Chaos, Solitons and Fractals, 2005; 26 : 1317-1335. 
[6] Wu YH, Han MA, Liu XL. On the study of limit cycles of a cubic polynomials system under Z4-equivariant quintic perturbation. Chaos, Solitons and Fractals, 2005; 24: 999-1012.

[7] Liu ZR, Li JB. Bifurcation set and distributions of limit cycles in Hamiltonian system approaching the principal deformation of Z-field. Int J Bifur Chaos, 1995; 3: 809-818.

[8] Zang H, Chen WC, Zhang TH. Perturbation from a cubic Hamiltonian with three figure eight-loops. Chaos, Solitons and Fractals, 2004; 22: 6174 .

[9] Li Jibin, Huang Qiming. Bifurcations of limit cycles forming compound eyes in the cubic system. Chinese Ann of Math, 1987, 8B (4): 391-403.

[10] Hong XC, Liu ZR. Fourteen limit cycles in a cubic Hamiltonian system with higher-order perturbed terms. Proceedings of the fourth international conference on nonlinear mechanics (Shanghai 2002): 1211-1215.

[11] Li JB, Liu ZR. Bifurcation set and limit cycles forming compound eyes in a perturbed Hamintonian system. Publ Math (Spain), 1991,35: 487-506.

[12] Tang MY, Hong XC. Fourteen limit cycles in a cubic Hamiltonian system with nine-order perturbed term. Chaos, Solitons and Fractals, 2002; 14:1361-1369.

[13] Zhang TH, Han MA, Zang H, Meng XZ. Bifurcation of limit cycles for a cubic Hamiltonian system under quartic perturbations. Chaos, Solitons and Fractals, 2004; 22: 1127-1138.

[14] Zhang TH, Zang H, Han MA. Bifurcation of limit cycles in a cubic system. Chaos, Solitons and Fractals, 2004; 20: 629-638.

[15] Li JB, Lin YP. Global bifurcations in a perturbed cubic system with Z2symmetry. Acta Mathematicae Applicatae Sinca, 1992; 8: 131-143.

[16] Huang W, Liu Y, Bifurcation of limit cycles from infinity for a class of quintic polynomial system, Bulletin DES Sciences Mathematiques, 2004; 128: 291-302.

[17] Zang H, Zhang T, Chan W, Bifurcation of limit cycles for quintic Hamiltonian systems with a double figure eight loop, J. Complexity, 2004; 20: 544-560. 
[18] Chen HB, Liu Y, Zeng X, center conditions and bifurcation of limit cycles at degenerate singular points in a quintic polynomial defferential system, Bulletin DES Sciences Mathematiques, 2005; 129: 127-138.

[19] Li CF, Li JB. Distribution of limit cycles for planar cubic Hamiltonian systems. Acta Nath Sinica 1985; 28: 509-521.

[20] Liu ZR, Qian TF, Li JB. Detection function method and its application to a perturbed quintic Hamiltonian system. Chaos, Solitons and Fractals, 2002; 13: 295-310.

[21] Helena EN, Yorke JA. Dynamics: numerical explorations (accompanying computer program dynamics co-authored by Eric J. Kostelich). New York: Springer; 1998.

Received: May 5, 2006 